\title{
Cómo Mejorar la Comprensión de los Riesgos Médicos en Personas Mayores
}

\section{How to Improve Comprehension of Medical Risks in Older Adults}

Recibido: Septiembre de 2012

Revisado: Febrero de 2013

Aceptado: Julio de 2013

\section{Rocio Garcia-Retamero, Rocio Muñoz}

Universidad de Granada, Espańa

Correspondencia

Rocio Garcia-Retamero, Ph.D. Facultad de Psicología, Universidad de Granada, Campus Universitario de Cartuja s/n, 18071 Granada, España, E-mail: rretamer@ugr. es. Este estudio ha sido financiado por el proyecto de investigación "How to Improve Understanding of Risks about Health (PSI2008-02019)" (Ministerio de Ciencia e Innovación; Espańa) y "Helping Doctors and Their Patients Make Decisions About Health (PSI2011-22954),” (Ministerio de Economía y Competitividad; España).

\section{Abstract}

Older adults frequently make decisions about their health. However, they prefer to play a passive role in this process. A plausible explanation of this result is that they often have difficulties understanding information about medical risks. In a study, we investigated the extent to what older adults $(n=136)$ understand information about the effectiveness of medical treatments. In particular, we compared accuracy of their inferences to that of young adults $(n=121)$. Participants were classified according to their graph literacy skills and received information about the effectiveness of medical treatments in a numerical or a visual format. Our study shows that visual aids substantially increased accuracy of inferences in older adults. Older adults with high graph literacy benefit most from the use of visual aids. We conclude that visual aids are often highly effective, transparent, and reliable risk communication tools. We discuss the practical implication of our results for the design of health campaigns.

Keywords: Aging, Visual Displays, Risk Perception, Information Dissemination, Visual Perception.

\section{Resumen}

Los mayores con frecuencia toman decisiones sobre su salud. A pesar de ello, prefieren adoptar un rol pasivo en este proceso. Una explicación que se ha ofrecido sobre este hecho es que frecuentemente presentan serias dificultades a la hora de comprender la información que reciben sobre los riesgos médicos. En un estudio, hemos investigado el grado en que los mayores $(n=136)$ comprenden la información sobre la efectividad de los tratamientos médicos. Concretamente, hemos comparado la precisión de las inferencias que éstos realizan con los juicios en adultos jóvenes $(n=121)$. Para ello, hemos presentado la información sobre la efectividad de los tratamientos en formato numérico o visual y hemos clasificado a los participantes según su nivel de habilidades gráficas. Nuestro estudio pone de manifiesto que la precisión de las inferencias que realizan los mayores se incrementa considerablemente cuando la información se representa visualmente. Esto ocurre especialmente cuando presentan habilidades gráficas elevadas. Concluimos que el uso de formatos visuales puede ser un método efectivo, transparente, y fiable de comunicación de la información sobre los riesgos médicos y la salud, y mencionamos las implicaciones prácticas de nuestros resultados para el desarrollo de campańas de salud.

Palabras claves: envejecimiento, medios visuales, percepción de riesgo, difusión de la información, percepción visual. 
Todos los pacientes tienen derecho a ser informados sobre los riesgos y beneficios derivados de los tratamientos médicos que reciben antes de acceder voluntariamente a los mismos. De este modo, pueden participar activamente en el proceso de toma de decisiones sobre su salud (Barry, 1999). Para ello, el personal sanitario ha de ser capaz de comunicar información relevante sobre dichos tratamientos, y los pacientes deben comprenderla y recordarla. Esta información se presenta habitualmente en probabilidades, porcentajes, o fracciones (Hanson, 2008). Así, por ejemplo, se sabe que la realización de mamografías reduce en un $25 \%$ la probabilidad de morir de cáncer de mama (Olsen et al., 2005), y que la realización del test de detección precoz del cáncer de próstata reduce en un $20 \%$ la probabilidad de morir a causa de esta enfermedad (Wilde, 2009). No obstante, diversos estudios han puesto de manifiesto que una proporción elevada de la población presenta serias limitaciones a la hora de comprender información numérica como la que aparece en estos ejemplos (Cokely, Galesic, Schulz, Ghazal, \& Garcia-Retamero, 2012; Galesic \& GarciaRetamero, 2010; Peters, 2012). Lo cual tiene consecuencias importantes, ya que con frecuencia muchos pacientes toman decisiones sesgadas sobre su salud (véase Reyna, Nelson, Han, \& Dieckmann, 2009 para una revisión).

Recientemente, se ha sugerido que el uso de ciertos formatos de presentación de la información, denominados formatos transparentes, puede mejorar la comunicación, la comprensión, y el recuerdo (Garcia-Retamero \& Cokely, en prensa a; Garcia-Retamero \& Galesic, 2013). El uso de formatos visuales es especialmente efectivo (Edwards, Elwyn, \& Mulley, 2002; Lipkus \& Peters, 2009; Paling, 2003). De hecho estos formatos pueden mejorar la comprensión de los riesgos asociados a diferentes pruebas diagnósticas, tratamientos médicos, y estilos de vida poco saludables (Ancker, Senathirajah, Kukafka, \& Starren, 2006; Galesic, Garcia-Retamero, \& Gigerenzer, 2009; Garcia-Retamero \& Hoffrage, 2013; Lipkus, 2007; Lipkus \& Hollands, 1999). También pueden promover la comprensión de los beneficios asociados a los tratamientos, aunque presenten efectos secundarios (Garcia-Retamero, Galesic, \& Gigerenzer, 2011; Waters, Weinstein, Colditz, \& Emmons, 2007; Zikmund-Fisher, Fagerlin, \& Ubel, 2008). Los formatos visuales de presentación de la información también pueden promover la realización de conductas saludables (GarciaRetamero \& Cokely, en prensa b), y pueden reducir los errores en los juicios inducidos por ejemplos anecdóticos o poco representativos (Cox, Cox, Sturm, \& Zimet,
2010; Fagerlin, Wang, \& Ubel, 2005), y los sesgos en la toma de decisiones (Garcia-Retamero \& Cokely, 2011, 2012; Garcia-Retamero \& Dhami, 2011, 2013; GarciaRetamero \& Galesic, 2009). Finalmente, la información visual sobre los riesgos médicos se comprende con más facilidad y en menos tiempo que la información presentada numéricamente (Goodyear-Smith et al., 2008).

Sin embargo, no todas las personas se benefician por igual del uso de formatos visuales de presentación de la información (Apter et al., 2008; Fagerlin, Ubel, Smith, \& Zikmund-Fisher, 2007; Peters, Hibbard, Slovic, \& Dieckmann, 2007). Se ha sugerido que estos formatos son especialmente útiles para comunicar información sobre la salud a personas con bajas habilidades numéricas (Galesic et al., 2009; Hawley et al., 2008; Peters et al., 2009), las cuales presentan habitualmente dificultades al procesar y utilizar conceptos numéricos básicos (Ancker \& Kaufman, 2007; Galesic \& Garcia-Retamero, 2010; Lipkus, \& Peters, 2009; Lipkus, Samsa, \& Rimer, 2001; Woloshin, Schwartz, Black, \& Welch, 1999). En contraste, las personas con altas habilidades numéricas suelen comprender esta información, incluso aunque no se presente visualmente (Garcia-Retamero \& Galesic, 2010).

Las personas también difieren en su nivel de habilidades gráficas (Galesic \& Garcia-Retamero, 2011), es decir, muestran distintos niveles en su capacidad para comprender y utilizar la información presentada visualmente- una habilidad que es independiente de las habilidades numéricas (Gaissmaier et al., 2012). En concreto, varios trabajos recientes han puesto de manifiesto que los formatos visuales son más beneficiosos para aquellas personas con niveles moderados o relativamente altos en sus habilidades gráficas. Por ejemplo, en un estudio realizado por Garcia-Retamero y Galesic (2010), se comprobó que la presentación de la información sobre la efectividad de un tratamiento médico mediante gráficas de barras o iconos puede ayudar a modificar las expectativas incorrectas de las personas. Este estudio además puso de manifiesto que las personas con niveles relativamente moderados o altos en sus habilidades gráficas son los que más se benefician del uso de los formatos visuales. Esto ocurrió a pesar de que tuvieran habilidades numéricas limitadas. Sin embargo, la representación visual de la información no resultó muy útil a las personas con bajas habilidades gráficas y numéricas. Estudios recientes realizados por Garcia-Retamero y Cokely (en prensa c) y Okan, Garcia-Retamero, Cokely, y Maldonado (2012) muestran resultados similares. 
El objetivo del presente estudio es investigar si los formatos visuales también pueden ser útiles para comunicar información sobre riesgos médicos a poblaciones vulnerables. Concretamente, nos hemos centrado en las personas mayores, ya que a menudo requieren una asistencia especial (Aceros, Cavalcante, \& Domènech, 2013; López \& Crespo, 2007). Debido al deterioro cognitivo relacionado con la edad, los mayores suelen tener problemas a la hora de comprender los riesgos sobre la salud cuando estos se presentan numéricamente (Park, 1999; Park \& Schwarz, 2002). De hecho, la evidencia empírica sugiere una relación directa entre la edad y las dificultades en la comprensión y el recuerdo de la información médica: Las personas mayores presentan más dificultades que los jóvenes, y estas dificultades se incrementan linealmente con el paso del tiempo (Finucane et al., 2002; Meyer, Russo, \& Talbot, 1995; Morrel, Park, \& Poon, 1990; Zwahr, Park, Eaton, \& Larson, 1997; Zwahr, Park, \& Shifren, 1999).

A pesar de estas dificultades, los mayores deben tomar decisiones sobre su salud con más frecuencia que los jóvenes (Park \& Schwarz, 2002). Los avances médicos y las mejoras en los estilos de vida han favorecido el incremento en la esperanza de vida de las personas en las últimas décadas (Organización Mundial de la Salud, 2012). Este incremento viene asociado a una mayor probabilidad de padecer diversas enfermedades crónicas y a un aumento progresivo en la necesidad de asistencia médica.

A pesar de todo ello, con frecuencia los mayores prefieren no desempeñar un rol activo en el proceso de toma de decisiones sobre su salud (Meyer, Talbot, \& Ranalli, 2007; véase Mather, 2006 para una revisión). Es decir, habitualmente presentan una tendencia a aceptar las sugerencias que les hace su médico sin cuestionarlas (Cassileth, Zupkis, SuttonSmith, \& March, 1980; Curley, Eraker, \& Yates, 1984). También solicitan menos información que les permita tomar dichas decisiones (Meyer et al., 1995), prefieren recibir un número menor de opciones o alternativas cuando toman las decisiones (Finucane et al., 2002; Reed, Mikels \& Simon, 2008; Zwahr et al., 1997), y utilizan estrategias heurísticas al tomarlas (Johnson, 1990, 1993; Park, 1999; Sanfey \& Hastie, 2000; Yates \& Patalano, 1999; véase Garcia-Retamero $\&$ Dieckmann, 2006 para una revisión). Por todo ello, los mayores requieren una ayuda y asistencia especial.

En este artículo, presentamos los resultados de un estudio dirigido a investigar el grado en que los mayores comprenden la información numérica sobre la efectividad de los tratamientos médicos. Concretamente, hemos comparado las inferencias que éstos hacen con los juicios realizados por adultos jóvenes. En nuestro estudio, hemos seguido el procedimiento empleado por Garcia-Retamero y Galesic (2010). Para ello, hemos presentado la información sobre la efectividad de los tratamientos en formato numérico o visual, y hemos clasificado a los participantes según su nivel de habilidades gráficas. También hemos medido el nivel de habilidades numéricas y el nivel educativo de los participantes para controlar su influencia en los resultados. Para incrementar la generalidad de nuestras conclusiones, hemos utilizado dos tipos de formatos visuales de presentación de la información sobre los riesgos médicos (gráficas de barras y de iconos). Partimos de la hipótesis de que los formatos visuales serían especialmente útiles para las personas mayores $\left(H_{1}\right)$, especialmente cuando presenten un nivel de habilidades gráficas relativamente alto $\left(H_{2}\right)$.

\section{Método}

\section{Participantes}

El estudio se ha realizado en una muestra de 121 adultos jóvenes y 136 mayores ( $\mathrm{N}$ total $=257$ ). Los adultos jóvenes proceden de las Universidades de Granada y Jaén, y tenían una edad comprendida entre los 19 y 40 años (Mediana = $32, D E=5.9)$. Todos ellos eran estudiantes universitarios de diversas disciplinas (p.ej., Psicología, Logopedia, Ingeniería). Los adultos mayores procedían de diversas residencias de Jaén y tenían una edad comprendida entre los 65 y los 93 años (Mediana $=70, D S=6.1$ ). La estructura de la muestra se describe en la Tabla 1. Los criterios de inclusión de los

\begin{tabular}{|c|c|c|c|c|}
\hline & \multicolumn{2}{|c|}{ Adultos jóvenes } & \multicolumn{2}{|c|}{ Adultos mayores } \\
\hline & $\mathrm{N}$ & $\begin{array}{c}\text { Porcen- } \\
\text { taje }\end{array}$ & $\mathrm{N}$ & $\begin{array}{c}\text { Porcen- } \\
\text { taje }\end{array}$ \\
\hline Total & 121 & 100 & 136 & 100 \\
\hline \multicolumn{5}{|l|}{ Género } \\
\hline Masculino & 57 & 47 & 62 & 46 \\
\hline Femenino & 64 & 53 & 74 & 54 \\
\hline \multicolumn{5}{|l|}{ Nivel educativo } \\
\hline Menos que secundaria & 0 & 0 & 19 & 14 \\
\hline Escuela secundaria & 0 & 0 & 100 & 74 \\
\hline Estudios universitarios & 121 & 100 & 17 & 12 \\
\hline
\end{tabular}


participantes en el estudio fueron presentar un nivel básico de lectura y escritura, tener 18 años o más (grupo de adultos jóvenes) o 65 años o más (grupo de adultos mayores), no tener problemas graves de visión y audición, no padecer enfermedades psiquiátricas o alcoholismo, y haber firmado el consentimiento informado.

\section{Materiales}

Los participantes completaron un cuestionario que incluía dos escalas de medida del nivel de habilidades gráficas y numéricas, respectivamente.

(I) Escala de habilidades gráficas. Consiste en trece ítems desarrollados por Galesic y Garcia-Retamero (2011) y mide tres habilidades (véase Friel, Curcio \& Bright, 2001): (1) la habilidad de comprensión de los datos, es decir, la capacidad para encontrar información específica en un gráfico (por ejemplo, la capacidad de deducir la altura de una barra dentro de una gráfica de barras, o el número de iconos de un tipo en una gráfica de iconos); (2) la habilidad de comprensión de las relaciones entre los datos, es decir, la capacidad para inferir relaciones entre los datos en un gráfico (por ejemplo, la capacidad de establecer la diferencia entre dos barras o conjuntos de iconos); y (3) la habilidad para realizar inferencias a partir de los datos, por ejemplo, la capacidad de proyectar una tendencia futura en una gráfica de líneas, y comprender la importancia de prestar atención a los rangos de la escala cuando se comparan dos gráficas. En el análisis de los datos, hemos dividido a los participantes en dos grupos de acuerdo a la puntuación media de su grupo de edad $(M=9.1, S E M=$ .24 en adultos jóvenes y $M=8.1, S E M=.24$ en mayores). El grupo de jóvenes con bajas habilidades gráficas incluye a aquellos participantes con 8 respuestas correctas o menos $(n=62,51 \%)$, mientras que el de jóvenes con altas habilidades gráficas incluye a los participantes con 9 respuestas correctas o más $(n=59,49 \%)$. El grupo de mayores con bajas habilidades gráficas incluye a aquellos participantes con 7 respuestas correctas o menos ( $\mathrm{n}=$ $66,49 \%)$, mientras que el grupo de mayores con altas habilidades gráficas incluye a quienes obtuvieron 8 respuestas correctas o más ( $\mathrm{n}=70,51 \%$; véase Gaissmaier et al., 2012; Garcia-Retamero \& Galesic, 2010 para un procedimiento similar).

(II) Escala de habilidades numéricas. Incluye nueve ítems y fue desarrollada y validada por Schwartz, Woloshin,
Black, y Welch (1997) y Lipkus et al. (2001). En el análisis de los datos, hemos controlado el efecto de las habilidades numéricas de los participantes. La media en la escala en los grupos de adultos jóvenes y mayores es $6(S E M=.2)$ y $5(S E M=.2)$, respectivamente.

Ambas escalas han mostrado una validez elevada en nuestro estudio ( $\alpha=.73$ para la escala de habilidades gráficas y $\alpha=.69$ para la escala de habilidades numéricas). Estos resultados son consistentes con los encontrados en un estudio dirigido a evaluar las propiedades psicométricas de las mismas en muestras representativas de participantes en cuanto a su edad, género, y nivel educativo en Estados Unidos y Alemania (véase Galesic \& Garcia-Retamero, 2010, 2011; Garcia-Retamero \& Galesic, 2010).

Una vez completadas las escalas, los participantes recibieron dos escenarios sobre la efectividad de Vitarilen, un fármaco ficticio para reducir el riesgo de infarto cerebral (escenario 1) e infarto de miocardio (escenario 2) en pacientes con síntomas de enfermedad arterial. En cada escenario, se describían los resultados de dos grupos de 100 pacientes seleccionados al azar que habían tomado Vitarilen o un placebo. El orden de presentación de los dos escenarios fue aleatorio.

\section{Diseño}

Hemos manipulado dos variables en un diseño mixto. En primer lugar, el nivel de reducción del riesgo relativo tras recibir el fármaco (es decir, la efectividad del tratamiento) se manipuló intra-sujetos con dos niveles: 25\% (escenario 1) y $75 \%$ (escenario 2 ). En el escenario 1, los participantes recibieron la siguiente información: "En comparación con el grupo de pacientes que ha tomado el placebo, la reducción del riesgo de sufrir un infarto cerebral en el grupo de pacientes que han ingerido Vitarilen es del 25\%." En el escenario 2, los participantes recibieron la siguiente información: "En comparación con el grupo de pacientes que ha tomado el placebo, la reducción del riesgo de sufrir un infarto de miocardio en el grupo de pacientes que han ingerido Vitarilen es del 75\%."

En segundo lugar, el formato del mensaje se ha manipulado entre-grupos con tres niveles. En un grupo, los participantes recibieron la información sobre el fármaco en formato numérico. En un segundo grupo, además de la información numérica, los participantes recibieron dos representaciones 
gráficas en forma de iconos (véase Figura 1). Dichas gráficas representaban el número de pacientes que habían sufrido el trastorno en el grupo que había ingerido el medicamento y el placebo, respectivamente. Los pacientes que habían sufrido el trastorno se representabanmediante círculos negros; los que no lo habían sufrido se representaban mediante círculos blancos. Los pacientes fueron representados mediante círculos debido a que en investigaciones previas se ha mostrado que no hay diferencias entre el uso de este tipo de material y otros estímulos menos abstractos (por ejemplo, caras representando a los pacientes; Gaissmaier et al., 2012; Stone, Yates, y Parker, 1997). En un tercer grupo, los participantes recibieron la información numérica y una representación gráfica formada por dos barras (véase Figura 2). En dicha gráfica se representaban el número de pacientes que habían sufrido el trastorno en el grupo que había ingerido el medicamento y el placebo respectivamente.

Finalmente se ha manipulado por selección la edad de los participantes y el nivel de habilidades gráficas, diferenciando entre un grupo de adultos jóvenes y otro de mayores, y un grupo de personas con bajas y altas habilidades gráficas en cada grupo de edad.

Como variable dependiente se ha medido la precisión en la comprensión de la información sobre el riesgo médico tras leer cada escenario. Siguiendo el procedimiento de Schwartz et al. (1997), los participantes primero estimaron cuántos pacientes sufrirían el trastorno (infarto cerebral o de miocardio) en un grupo de 1.000 pacientes con síntomas de enfermedad arterial "si no toman" el medicamento. En segundo lugar, los participantes estimaron cuántos pacientes sufrían el trastorno (infarto cerebral o de miocardio) en un grupo de 1.000 pacientes con síntomas de enfermedad

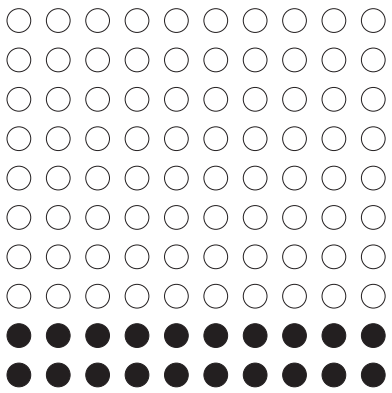

Placebo

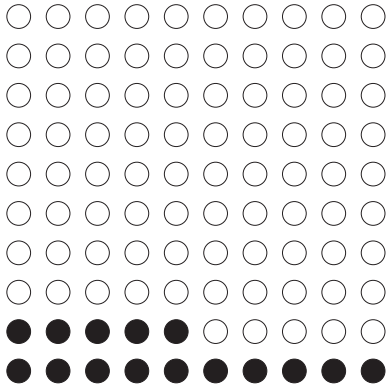

Vitarilen
Figura 1. Representación gráfica de iconos presentada junto a la información numérica sobre la reducción del riesgo de padecer el trastorno (escenario 1 en la condición información numérica e iconos).

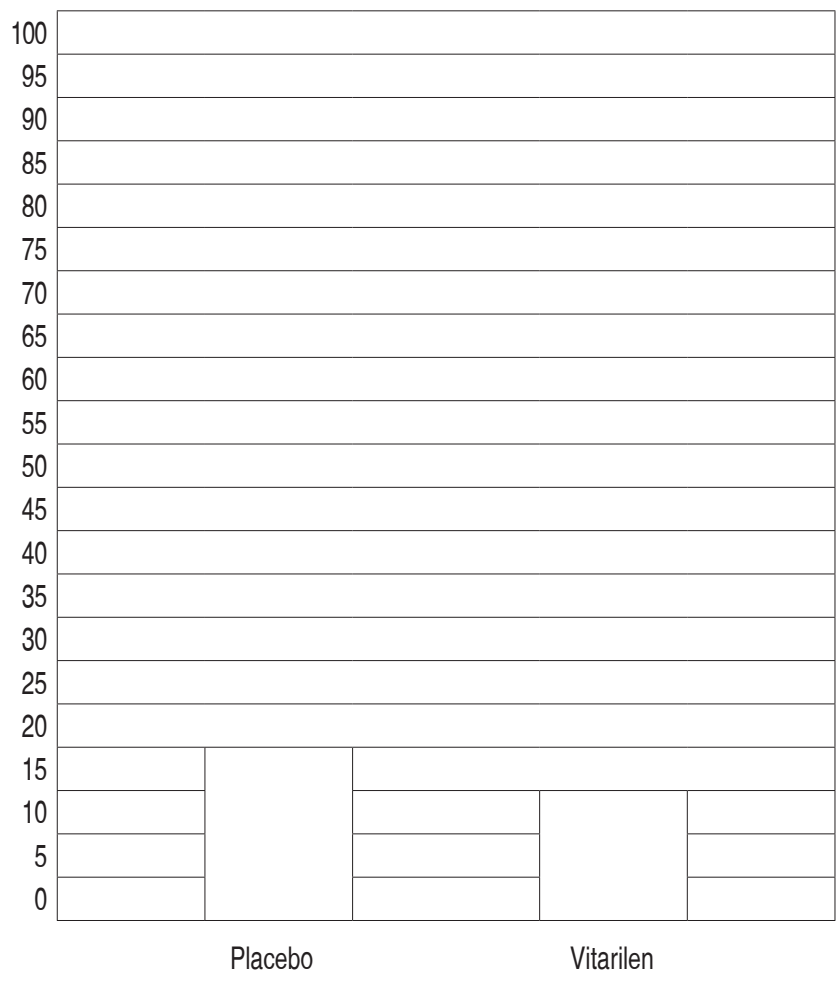

Figura 2. Representación gráfica de barras presentada junto a información numérica sobre la reducción del riesgo de padecer el trastorno (escenario 1 en la condición información numérica y barras).

arterial "si toman" el medicamento. La reducción de riesgo relativo de cada paciente se obtuvo restando la respuesta obtenida en la segunda pregunta a la obtenida en la primera, y dividiendo el resultado por la primera (véase Schwartz et al., 1997). Siguiendo el procedimiento de Garcia-Retamero y Galesic (2010), se ha considerado que los participantes realizaban una estimación correcta cuando proporcionaban una respuesta correcta en al menos uno de los dos escenarios (es decir, en uno de ellos o en ambos).

\section{Procedimiento}

La recogida de datos se realizó entre Noviembre de 2011 y Abril de 2012. Los participantes contestaron un cuestionario de papel y lápiz, invirtiendo en ello 15 minutos en promedio. $\mathrm{Al}$ comienzo del estudio, todos los participantes firmaron una hoja de consentimiento para participar en el estudio. Al finalizar el estudio, proporcionaron sus datos demográficos. El comité ético de la Universidad de Granada aprobó la metodología empleada en este estudio. 


\section{Análisis de datos}

Hemos realizado un análisis de regresión logística para evaluar el efecto del formato del mensaje (numérico vs. numérico e iconos vs. numérico y barras) y la interacción de este factor con el nivel de habilidades gráficas (altas vs. bajas) y la edad (adultos jóvenes vs. adultos mayores) sobre el porcentaje de participantes que han proporcionado una estimación correcta en al menos una de los dos escenarios. Hemos controlado el efecto del nivel de habilidades numéricas y del nivel educativo de los participantes, incluyendo ambos factores como variables continuas en el análisis.

\section{Resultados}

Cuando se proporcionó la información en formato numérico, el 53\% (error estándar $[\mathrm{EE}]=4$ ) de los participantes realizó una estimación correcta sobre la efectividad del tratamiento en al menos uno de los dos escenarios. En cambio, cuando se añadió la gráfica de iconos o barras a la información numérica, el $62 \%(\mathrm{EE}=4.7)$ y el $64 \%(\mathrm{EE}=5)$ de los participantes, respectivamente, proporcionó una estimación correcta sobre la reducción del riesgo en al menos uno de los dos escenarios. En línea con estos resultados, el análisis de regresión logística ha mostrado un efecto principal significativo de la variable formato del mensaje [estadístico de Wald $(1)=4.18, p=.04]$.

Los adultos jóvenes en general proporcionaron una respuesta correcta en al menos uno de los escenarios con más frecuencia que los mayores $(74 \%, \mathrm{EE}=4$ vs. $49 \%$, $\mathrm{EE}=4.3$ ). Además, las personas con altas habilidades gráficas (independiente de su edad) se benefician en mayor medida del material visual de apoyo que las personas con habilidades gráficas bajas $(75 \%, \mathrm{EE}=3.8$ vs. $46 \%, \mathrm{EE}=$ 4.4; véase Figura 3). En línea con estos resultados, el análisis de regresión logística ha mostrado un efecto principal significativo de la variable edad [estadístico de Wald (1)= 9.84, $p=.002]$ y una interacción entre el formato del mensaje y el nivel de habilidades gráficas [estadístico de Wald $(1)=8.18, p=.004)$. Ninguna otra interacción ha resultado significativa (véase Tabla 2).

\section{Discusión}

Las personas mayores suelen tomar decisiones sobre su salud con más frecuencia que los jóvenes (Park \& Schwarz, 2002). A pesar de ello, los mayores prefieren adoptar un

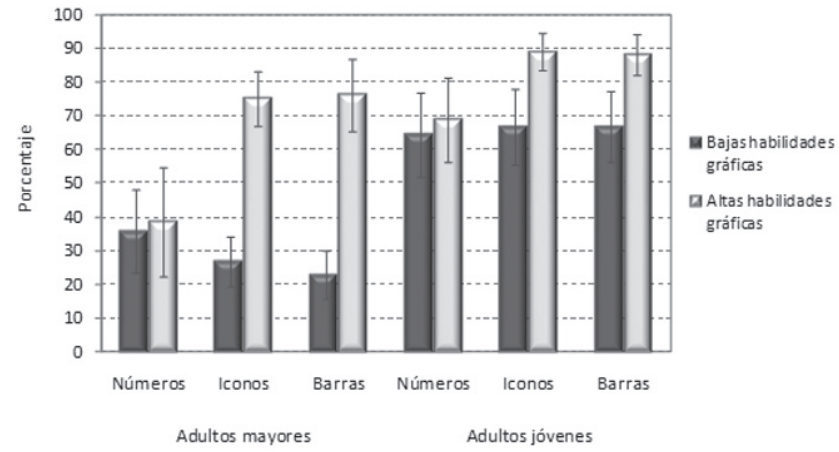

Figura 3. Porcentaje de participantes con altas y bajas habilidades gráficas que proporcionó una estimación correcta sobre la efectividad del tratamiento en al menos uno de los escenarios en función de la edad y del formato del mensaje. Las barras de error representan un error estándar.

Nota: Iconos = Información numérica e iconos. Barras = Información numérica y barras.

\begin{tabular}{|c|c|c|}
\hline & Estadístico de Wald & $P$ \\
\hline Habilidades numéricas & 20.25 & $.0001^{*}$ \\
\hline Nivel educativo & 1.64 & .200 \\
\hline Edad (1) & 9.84 & $.002^{*}$ \\
\hline Formato del mensaje (2) & 4.18 & $.041^{*}$ \\
\hline Habilidades gráficas (3) & .01 & .920 \\
\hline $1 \times 2$ & .030 & .855 \\
\hline $1 \times 3$ & .29 & .588 \\
\hline $2 \times 3$ & 8.18 & $.004^{*}$ \\
\hline $1 \times 2 \times 3$ & 1.36 & .243 \\
\hline
\end{tabular}

rol pasivo en este proceso (Meyer et al., 2007). Una de las posibles explicaciones que se han ofrecido sobre este hecho es que frecuentemente muestran problemas severos a la hora de comprender y utilizar la información que reciben sobre los riesgos médicos (Gigerenzer, Gaissmaier, Kurz-Milcke, Schwartz \& Woloshin, 2007), lo cual les hace vulnerables a los problemas de salud. Los resultados de nuestro estudio apoyan esta idea y ponen de manifiesto que las personas mayores también muestran limitaciones a la hora de inferir correctamente la efectividad de los tratamientos médicos. 
Los resultados anteriormente presentados son coherentes con diversos estudios que muestran que la edad está asociada a un deterioro cognitivo progresivo que afecta a los juicios y la toma de decisiones (p. ej., un deterioro en el control y la regulación de las cogniciones; Amieva, Phillips, \& Della Sala, 2003). En este sentido, se ha comprobado que las personas mayores procesan la información con mayor lentitud que los jóvenes (Salthouse, 1992, 1994), tienen más dificultades a la hora de inhibir el efecto de la información irrelevante en una tarea (Hasher \& Zacks, 1988), y muestran más déficits de aprendizaje (Cohen, 1996; Kausler, 1990; Salthouse, McGuthry, \& Hambrick, 1999). Los mayores también presentan una menor precisión en sus estimaciones sobre las frecuencias numéricas absolutas (Mutter \& Goedert, 1997), son menos conscientes que los jóvenes de los factores que influyen en sus decisiones (Lopatto et al., 1998), y muestran un exceso de confianza en la precisión de sus inferencias (Crawford \& Stankov, 1996) y una menor capacidad a la hora de controlar el impacto de los procesos automáticos en sus juicios (Hess, McGee, Woodburn, \& Bolstad, 1998; Hess, Waters, \& Bolstad, 2000). Todo ello afecta a la precisión de sus decisiones (Peters, Hess, Västfjäll, \& Auman, 2007), incluyendo aquellas relativas a su salud y a los tratamientos médicos que reciben.

No obstante, nuestro estudio pone de manifiesto el hecho de que hay métodos que pueden facilitar la comprensión de la información sobre los riesgos médicos. En línea con nuestra hipótesis $\left(H_{1}\right)$, la representación visual de la información sobre la efectividad de los tratamientos mejora considerablemente la precisión de las inferencias que realizan las personas mayores. Los formatos visuales también mejoran la ejecución en adultos jóvenes, aunque en menor medida, lo cual es congruente con la investigación previa (véase Garcia-Retamero \& Galesic, 2009, 2010). Esta mejora se hace notable de manera especial en el grupo de personas mayores y jóvenes con altas habilidades gráficas $\left(\mathrm{H}_{2}\right)$. Nuestro estudio, por tanto, muestra que la escala de habilidades gráficas diseñada por Galesic y GarciaRetamero (2011) permite identificar aquellas personas que se benefician en mayor medida de la representación visual de la información numérica sobre la salud. Es probable que haya que diseñar otro tipo de formatos para comunicar los riesgos médicos a los pacientes con bajas habilidades gráficas. En este sentido, el uso de analogías y/o el entrenamiento adicional en la comprensión de información gráfica puede ser una estrategia apropiada
(Galesic \& Garcia-Retamero, 2013; véase también Pérez Echeverría, Postigo, \& Pecharromán, 2008).

Finalmente, nuestros resultados son consistentes con la investigación que sugiere que los formatos visuales de presentación de la información permiten codificar la información relevante con mayor probabilidad (GarciaRetamero \& Cokely, 2011; véase también Spiegelhalter, Pearson, \& Short, 2011) y comprender la relación entre clases (ej., el número total de personas que han recibido un tratamiento y el número de personas que habiendo recibido el tratamiento muestran una enfermedad; Reyna $\&$ Brainerd, 2008). En definitiva, nuestros datos apoyan la hipótesis de que muchos de los problemas que se producen en la comprensión y la comunicación de la información sobre riesgos se deben en parte al modo en que se representa el problema que han de resolver (Gigerenzer \& Edwards, 2003; Gigerenzer et al., 2007). Nuestros datos además muestran que el uso de formatos inadecuados de presentación de la información puede interactuar con las habilidades y las características de las personas que los interpretan (p. ej., la edad). Mejoras importantes en la comprensión de la información y reducción en lo que aparentemente se ha interpretado como pensamientos sesgados se han encontrado también en otros ámbitos como el razonamiento con probabilidades condicionadas cuando se expresan como frecuencias naturales (Gigerenzer \& Hoffrage, 1995), la percepción sobre la reducción de riesgos relativos cuando se expresan en términos absolutos (Covey, 2007). Con nuestra investigación, vamos un paso más allá generalizando estas conclusiones a las estimaciones sobre la efectividad de los tratamientos en personas mayores.

La escala diseñada por Galesic y Garcia-Retamero (2011) es breve y ha mostrado buenas propiedades psicométricas (véase Garcia-Retamero \& Galesic, 2010). Nuestra investigación sugiere que su uso con fines clínicos o de investigación puede ser apropiado. De hecho, podría utilizarse exclusivamente alguna de las subescalas que la componen en situaciones con poca disponibilidad de tiempo. En este sentido, una posible vía para investigación futura podría ser la aplicación de la escala en personal médico de distintas edades y con distinto grado de experiencia. Diversos estudios recientes sobre habilidades numéricas y toma de decisiones sobre la salud han mostrado que no solo los pacientes mayores presentan problemas para comprender conceptos numéricos esenciales; sus médicos muestran los mismos problemas (Gigerenzer et al., 2007). Sin embargo, 
las diferencias entre pacientes y personal sanitario en sus habilidades para la comprensión de información visual aún no han sido objeto de estudio. En investigaciones futuras también se podría poner a prueba si la escala diseñada por Galesic y Garcia-Retamero (2011) permite medir las habilidades de comprensión de información sobre riesgos en áreas diferentes a la salud (p. ej., finanzas, nutrición, o educación). Aunque los resultados en nuestro trabajo permiten obtener conclusiones claras, es posible que haya diferencias sustanciales en las percepciones sobre los riesgos en distintos ámbitos.

También sería interesante replicar nuestros datos en distintas muestras de participantes. Los grupos de participantes en nuestro estudio no sólo diferían en cuanto a su edad. El grupo de participantes jóvenes además tenía un nivel educativo más elevado. Asimismo, el rango de edad y la varianza de la edad eran más amplios en el grupo de personas mayores que en el de jóvenes. Aunque hemos controlado las diferencias en el nivel educativo en nuestros análisis estadísticos, los trabajos futuros podrían determinar la generalidad de nuestras conclusiones empleando un grupo de control más similar al de adultos mayores. Finalmente, nuestros resultados sugieren que podría ser útil emplear formatos visuales de presentación de la información en las campañas dirigidas a prevenir hábitos poco saludables (ej., fumar, ingesta masiva de alcohol) o a promover la detección precoz de diversas enfermedades (ej., cáncer de mama o próstata). Nuestros hallazgos han resultado prometedores y sugieren que pueden incrementar la efectividad de dichas campañas.

\section{Referencias}

Aceros, J. C., Cavalcante, M. T., \& Domènech, M. (2013). Identidad de lugar en usuarios de teleasistencia: Un análisis conversacional. Revista Latinoamericana de Psicologia, 45, 81-95. Disponible en: http:// openjournal.konradlorenz.edu.co/index.php/rlpsi/ article/view/1316/768

Amieva, H., Phillips, L., \& Della Sala, S. (2003). Behavioral dysexecutive symptoms in normal aging. Brain and Cognition, 53, 129-132. doi:10.1016/S02782626(03)00094-0.

Ancker, J. S., \& Kaufman, D. (2007). Rethinking health numeracy: A multidisciplinary literature review. Journal of the American Medical Informatics Association, 14, 713-721.
Ancker, J. S., Senathirajah, Y., Kukafka, R., \& Starren, J. B. (2006). Design features of graphs in health risk communication: A systematic review. Journal of the American Medical Informatics Association, 13, 608-618.

Apter, A. J., Paasche-Orlow, M. K., Remillard, J. T., Bennett, I. M., Ben-Joseph, E. P., Batista, R. M., Hyde, J., \& Rudd, R. E. (2008). Numeracy and communication with patients: They are counting on us. Journal of General Internal Medicine, 23, 2117-2124. Doi: 10.1007/s11606-008-0803-x

Barry, M. D. (1999). Involving patients in medical decisions. Journal of the American Medical Association, 282, 2356-2357. doi:10.1001/jama.282.24.2356

Cassileth, B. R., Zupkis, R. V., Sutton-Smith, K., \& March, V. (1980). Information and participation preferences among cancer patients. Annals of Internal Medicine, 2, 832-836.

Cohen, G. (1996). Memory and learning in normal aging. En R. T. Woods (Ed.), Handbook of the clinical psychology of aging (pp. 43-58). Chichester, England: Wiley.

Cokely, E. T., Galesic, M., Schulz, E., Ghazal, S., \& Garcia-Retamero, R. (2012). Measuring risk literacy: The Berlin Numeracy Test. Judgment and Decision Making, 7, 25-47.

Covey, J. A. (2007). Meta-analysis of the effects of presenting treatment benefits in different formats. Medical Decision Making, 27, 638-654. Doi: 10.1177/0272989X07306783

Cox, D. S., Cox, A. D., Sturm, L., \& Zimet, G. (2010). Behavioral interventions to increase $\mathrm{HPV}$ vaccination acceptability among mothers of young girls. Health Psychology, 29, 29-39. doi: 10.1037/a0016942

Crawford, J. D., \& Stankov, L. (1996). Age differences in the realism of confidence judgments: A calibration study using tests of fluid and crystallized intelligence. Learning and Individual Differences, 8, 83-103. doi: http://dx.doi.org/10.1016/S1041-6080(96)90027-8

Curley, S. P., Eraker, S. A., \& Yates, J. F. (1984). An investigation of patients' reactions to therapeutic uncertainty. Medical Decision Making, 4, 501-511.

Edwards, A., Elwyn, G., \& Mulley, A. (2002). Explaining risks: Turning numerical data into meaningful pictures. British Medical Journal, 324, 827-830. doi: http://dx.doi.org/10.1136/bmj.324.7341.827 
Fagerlin, A., Ubel, P. A., Smith, D. M., \& Zikmund-Fisher, B. J. (2007). Making numbers matter: Present and future research in risk communication. American Journal of Health Behavior, 31, S47-S56. doi:10.5555/ ajhb.2007.31.supp.S47

Fagerlin, A., Wang, C., \& Ubel, P. A. (2005). Reducing the influence of anecdotal reasoning on people's health care decisions: Is a picture worth a thousand statistics? Medical Decision Making, 25, 398-405. doi: 10.1177/0272989X05278931.

Finucane, M. L., Slovic, P., Hibbard, J. H., Peters, E., Mertz, C. K., \& Macgregor, D. G. (2002). Aging and decision-making competence: An analysis of comprehension and consistency skills in older versus younger adults considering health-plan options. Journal of Behavioral Decision Making, 15, 141-164. doi: 10.1002/bdm.40

Friel, S. N., Curcio, F. R., \& Bright, G. W. (2001). Making sense of graphs: critical factors influencing comprehension and instructional implications. Journal for Research in Mathematics Education, 32, 124-158. Disponible en: http://www.jstor.org/stable/749671.

Gaissmaier, W., Wegwarth, O., Skopec, D., Müller, A. S., Broschinski, S., \& Politi, M. C. (2012). Numbers can be worth a thousand pictures: Individual differences in understanding graphical and numerical representations of health-related information. Health Psychology, 31, 286-296. doi: 10.1037/a0024850

Galesic, M., \& Garcia-Retamero, R. (2010). Statistical numeracy for health: A cross-cultural comparison with probabilistic national samples. Archives of Internal Medicine, 170, 462-468. doi: 10.1001/ archinternmed.2009.481

Galesic, M., \& Garcia-Retamero, R. (2011). Graph literacy: A cross-cultural comparison. Medical Decision Making, 31, 444-457. doi: 10.1177/0272989X10373805

Galesic, M., \& Garcia-Retamero, R. (2013). Using analogies to communicate information about health risks. Applied Cognitive Psychology, 27, 33-42. doi: 10.1002/ acp. 2866

Galesic, M., Garcia-Retamero, R., \& Gigerenzer, G. (2009). Using icon arrays to communicate medical risks: Overcoming low numeracy. Health Psychology, 28, 210-216. doi: 10.1037/a0014474

Garcia-Retamero, R., \& Cokely, E. T. (2011). Effective communication of risks to young adults: Using message framing and visual aids to increase condom use and STD screening. Journal of Experimental Psychology: Applied, 17, 270-287. doi: 10.1037/a0023677.

Garcia-Retamero, R., \& Cokely, E. T. (2012). Advances in efficient health communication: Promoting prevention and detection of STDs. Current HIV Research, 10, 262-270. doi: http://dx.doi. org/10.2174/157016212800618084.

Garcia-Retamero, R., \& Cokely, E. (en prensa a). Communicating health risks with visual aids. Current Directions in Psychological Science. doi: 10.1177/0963721413491570

Garcia-Retamero, R., \& Cokely, E. T. (in press b). Simple but powerful health messages for increasing condom use in young adults. Journal of Sex Research. DOI: 10.1080/00224499.2013.806647

Garcia-Retamero, R., \& Cokely, E. (en prensa c). The influence of skills, message frame, and visual aids on prevention of sexually transmitted diseases. Journal of Behavioral Decision Making. doi:10.1002/bdm.1797

Garcia-Retamero, R., \& Dhami, M. K. (2011). Pictures speak louder than numbers: On communicating medical risks to immigrants with limited non-native language proficiency. Health Expectations, 14, 46-57. doi: 10.1111/j.1369-7625.2011.00670.x

Garcia-Retamero, R., \& Dhami, M. K. (2013). On avoiding framing effects in experienced decision makers. The Quarterly Journal of Experimental Psychology, 66, 829-842. doi: 10.1080/17470218.2012.727836

Garcia-Retamero, R., \& Dieckmann, A. (2006). Una visión crítica del enfoque de los heurísticos rápidos y frugales. Revista Latinoamericana de Psicología, 38, 509-522.Disponible en: http://www.scielo.org.co/ pdf/rlps/v38n3/v38n3a05.pdf

Garcia-Retamero, R., \& Galesic, M. (2009). Communicating treatment risk reduction to people with low numeracy skills: A cross-cultural comparison. American Journal of Public Health, 99, 2196-2202. doi: 10.2105/ AJPH.2009.160234

Garcia-Retamero, R., \& Galesic, M. (2010). Who profits from visual aids: Overcoming challenges in people's understanding of risks. Social Science \& Medicine, 70, 1019-1025. doi: 10.1016/j.socscimed.2009.11.031

Garcia-Retamero, R., \& Galesic, M. (2013). Transparent communication of health risks: Overcoming cultural differences. New York, NY: Springer. 
Garcia-Retamero, R., Galesic, M., \& Gigerenzer, G. (2011). Cómo favorecer la comprensión y la comunicación de los riesgos sobre la salud. Psicothema, 23, 599-605. Disponible en: http://library.mpib-berlin.mpg.de/ $\mathrm{ft} / \mathrm{rg} / \mathrm{RG}$ _Como_2011.pdf

Garcia-Retamero, R., \& Hoffrage, U. (2013). Visual representation of statistical information improves diagnostic inferences in doctors and their patients. Social Science and Medicine, 83, 27-33.

Gigerenzer, G., \& Edwards, A. (2003). Simple tools for understanding risks: From innumeracy to insight. British Medical Journal, 327, 741-744. doi: http:// dx.doi.org/10.1136/bmj.327.7417.741

Gigerenzer, G., Gaissmaier, W., Kurz-Milcke, E., Schwartz, L. M., \& Woloshin, S. (2007). Helping doctors and patients make sense of health statistics. Psychological Science in the Public Interest, 8, 53-96.

Gigerenzer, G., \& Hoffrage, U. (1995). How to improve Bayesian reasoning without instruction: Frequency formats. Psychological Review, 102, 684-704.

Goodyear-Smith, F., Arroll, B., Chan, L., Jackson, R., Wells, S., \& Kenealy, T. (2008). Patients prefer pictures to numbers to express cardiovascular benefit from treatment. Annals of Family Medicine, 6, 213-217. doi: 10.1370/afm.795.

Hanson, J. L. (2008). Shared decision making: Have we missed the obvious? Archives of Internal Medicine, 168, 1368-1370. doi:10.1001/archinte.168.13.1368

Hasher, L., \& Zacks, R. T. (1988). Working memory, comprehension, and aging: A review and a new view. En G. H. Bower (Ed.), The psychology of learning and motivation: Advances in research and theory (Vol. 22, pp. 193-225). San Diego, CA: Academic Press.

Hawley, S. T., Zikmund-Fisher, B., Ubel, P., Jancovic, A., Lucas, T., \& Fagerlin, A. (2008). The impact of the format of graphical presentation on health-related knowledge and treatment choices. Patient Education and Counseling, 73, 448-455. doi: 10.1016/j. pec.2008.07.023

Hess, T. M., McGee, K. A., Woodburn, S. M., \& Bolstad, C. A. (1998). Age-related priming effects in social judgments. Psychology and Aging, 13, 127-137.

Hess, T. M., Waters, S. J., \& Bolstad, C. A. (2000). Motivational and cognitive influences on affective priming in adulthood. Journal of Gerontology: Psychological Sciences, 55B, 193-204.
Johnson, M. M. S. (1990). Age differences in decision making: A process methodology for examining strategic information processing. Journal of Gerontology Psychological Sciences, 45, 75-78. doi: 10.1093/geronj/45.2.P75

Johnson, M. M. S. (1993). Thinking about strategies during, before, and after making a decision. Psychology and Aging, 8, 231-241.

Kausler, D. H. (1990). Automaticity of encoding and episodic memory processes. En E. A. Lovelace (Ed.), Aging and cognition: Mental processes, self-awareness, and interventions. Advances in psychology (vol. 72, pp. 29-67). Amsterdam: North-Holland.

Lipkus, I. M. (2007). Numeric, verbal, and visual formats of conveying health risks: Suggested best practices and future recommendations. Medical Decision Making, 27, 696-713. doi: 10.1177/0272989X07307271.

Lipkus, I. M., \& Hollands, J. G. (1999). The visual communication of risk. JNCI Monographs, 25, 149-163.

Lipkus, I. M., \& Peters, E. (2009). Framework and practical insights understanding the role of numeracy in health: Proposed theoretical insights. Health Education \& Behavior, 36, 1065-1081. doi: 10.1177/1090198109341533.

Lipkus, I. M., Samsa, G., \& Rimer, B. K. (2001). General performance on a numeracy scale among highly educated samples. Medical Decision Making, 21, $37-44$.

Lopatto, D. E., Ogier, S., Wickelgren, E. A., Gibbens, C., Smith, A., Sullivan, L., \& Muns, M. (1998). Cautiousness, stereotypy, and variability in older and younger adults. Psychological Record, 48, 571589. Disponible en: http://opensiuc.lib.siu.edu/tpr/

López, J., \& Crespo, M. (2007). Intervenciones con cuidadores de familiares mayores dependientes: Una revisión. Psicothema, 19, 72-80. Disponible en: http://www.psicothema.com/pdf/3330.pdf.

Mather, M. (2006). A review of decision making processes: Weighing the risks and benefits of aging. En L. L. Carstensen \& C. R. Hartel (Eds.), When I'm 64 (pp. 145-173). Washington, DC: National Academies Press.

Meyer, B. J. F., Russo, C., \& Talbot, A. (1995). Discourse comprehension and problem solving: Decisions about the treatment of breast cancer by women across the lifespan. Psychology and Aging, 10, 84-103. 
Meyer, B. J. F., Talbot, A. P., \& Ranalli, C. K. (2007). Why older adults make more immediate treatment decisions about cancer than younger adults. Psychology and Aging, 22, 505-524. doi: 10.1037/08827974.22.3.505

Morrell, R. W., Park, D. C., \& Poon, L. W. (1990). Effects of labeling techniques on memory and comprehension of prescription information in young and older adults. Journal of Gerontology: Psychological Sciences, 45, 166-172. doi: 10.1093/geronj/45.4.P166

Mutter, S. A., \& Goedert, K. M. (1997). Frequency discrimination vs. frequency estimation: Adult age differences and the effect of divided attention. Journal of Gerontology: Psychological Sciences, 52B, 319-328.

Okan, Y., Garcia-Retamero, R., Cokely, E. T., \& Maldonado, A. (2012). Individual differences in graph literacy: Overcoming denominator neglect in risk comprehension. Journal of Behavioral Decision Making, 25, 390-401. doi: 10.1002/bdm.751

Olsen, A. H., Njor, S. H., Vejborg, I., Schwartz, W., Dalgaard, P., Jensen, M., Tange, U. B., BlichertToft, M., Rank, F., Mouridsen, H., \& Lynge, E. (2005). Breast cancer mortality in Copenhagen after introduction of mammography screening: Cohort study. British Medical Journal, 330, 220-224. doi: 10.1136/bmj.38313.639236.82

Organización Mundial de la Salud (2012). Diez datos sobre el envejecimiento de la población. Disponible en http://www.who.int/features/factfiles/ageing/es/

Paling, J. (2003). Strategies to help patients understand risks. British Medical Journal, 327, 745-748. doi:10.1136/ bmj.327.7417.745

Park, D. C. (1999). Aging and the controlled and automatic processing of medical information and medical intentions. En D. C. Park, R. W. Morrell, \& K. Shifren (Eds.), Processing of medical information in aging patients: Cognitive and human factors perspectives (pp. 3-22). Mahwah, NJ: Lawrence Erlbaum Associates.

Park, D., \& Schwarz, N. (2002). Envejecimiento cognitivo. Madrid: Médica Panamericana.

Pérez Echeverría, M. P., Postigo, Y., \& Pecharromán, A. (2008). Graphicacy: University students' skills in translating information. En C. Andersen, N. Scheuer, M. P. Pérez Echeverría, \& E. Teubal (Eds.),
Representational systems and practices as learning tools (pp. 209-224). Rotterdam: Sense Publishers.

Peters, E. (2012). Beyond comprehension: The role of numeracy in judgment and decision making. Current Directions in Psychological Science, 21, 31-35. doi: 10.1177/0963721411429960

Peters, E., Dieckmann, N. F., Västfäll, D., Mertz, C. K., Slovic, P., \& Hibbard, J. (2009). Bringing meaning to numbers: The impact of evaluative categories on decisions. Journal of Experimental Psychology Applied, 15, 213-227. doi: 10.1037/a0016978

Peters, E., Hess, T. M., Västfjäll, D., \& Auman, C. (2007). Adult age differences in dual information processes: Implications for the role of affective and deliberative processes in older adults' decision making. Perspectives on Psychological Science, 2, 1-23. doi: 10.1002 lencr. 23944

Peters, E., Hibbard, J., Slovic, P., \& Dieckmann, N. (2007). Numeracy skill and the communication, comprehension, and use of risk-benefit information. Health Affairs, 26, 741-748. doi: 10.1377/ hlthaff.26.3.741

Reed, A. E., Mikels, J. A, \& Simon, K. I. (2008). Older adults prefer less choice than young adults. Psychology and aging, 23, 671-575. doi: 10.1037/a0012772

Reyna, V. F., \& Brainerd, C. J. (2008). Numeracy, ratio bias, and denominator neglect in judgments of risk and probability. Learning and Individual Differences, 18, 89-107. doi:http://dx.doi.org/10.1016/j. lindif.2007.03.011

Reyna, V. F., Nelson, W. L., Han, P. K., \& Dieckmann, N. F. (2009). How numeracy influences risk comprehension and medical decision making. Psychological Bulletin, 135, 943-973. doi: 10.1037/ a0017327

Salthouse, T. A. (1992). Why do adult age differences increase with task complexity? Developmental Psychology, 28, 905-918. doi: 10.1037/0012-1649.28.5.905

Salthouse, T. A. (1994). The nature of the influence of speed on adult age differences in cognition. Developmental Psychology, 30, 240-259.

Salthouse, T. A., McGuthry, K. E., \& Hambrick, D. Z. (1999). A framework for analyzing and interpreting differential aging pat-terns: Application to three measures of implicit learning. Aging, Neuropsychology, and Cognition, 6, 1-18. doi:10.1076/anec.6.1.1.789. 
Sanfey, A., \& Hastie, R. (2000). Judgment and decision making across the adult life span: A tutorial review of psychological research. En D. Park \& N. Schwarz (Eds.), Aging and cognition: A primer (pp. 253-273). Philadelphia: Psychology Press.

Schwartz, L. M., Woloshin, S., Black, W. C., \& Welch, H. G. (1997). The role of numeracy in understanding the benefit of screening mammography. Annals of Internal Medicine, 127, 966-972.

Spiegelhalter, D., Pearson, M., \& Short, I. (2011). Visualizing uncertainty about the future. Science, 333, 1393-1400. doi: 10.1126/science. 1191181

Stone, E. R., Yates, J. F., \& Parker, A. M. (1997). Effects of numerical and graphical displays on professed risktaking behavior. Journal of Experimental Psychology: Applied, 3, 243-256.

Waters, E. A., Weinstein, N. D., Colditz, G. A., \& Emmons, K. M. (2007). Reducing aversion to side effects in preventive medical treatment decisions. Journal of Experimental Psychology: Applied, 13, 11-21.

Wilde J. (2009). PSA screening cuts deaths by $20 \%$, says world's largest prostate cancer study. ERSPC Press
Office, Carver Wilde Communications. Disponible en: http://www.erspc-media.org/release090318.php.

Woloshin, S., Schwartz, L. M., Black, W. C., \& Welch, H. G. (1999). Women's perceptions of breast cancer risk: How you ask matters. Medical Decision Making, 19, 221-229.

Yates, J. F., \& Patalano, A. L. (1999). Decision making and aging. En D. C. Park \& R. W. Morrell (Eds.) Processing of medical information in aging patients (pp. 31-54). Mahwah, NJ: Erlbaum.

Zikmund-Fisher, B. J., Fagerlin, A., \& Ubel, P. A. (2008). Improving understanding of adjuvant therapy options by using simpler risk graphics. Cancer, 113, 3382-3390. doi: 10.1002/cncr.23959

Zwahr, M. D., Park, D. C., Eaton, T. A., \& Larson, E. J. (1997). Implementation of the patient selfdetermination act: A comparison of nursing homes to hospitals. Journal of Applied Gerontology, 16, 190-207.

Zwahr, M. D., Park, D. C., \& Shifren, K. (1999). Judgments about estrogen replacement therapy: The role of age, cognitive abilities, and beliefs. Psychology and Aging, 14, 179-191. 